\title{
Salud e interculturalidad: Sugerencias para organizaciones de salud en contextos indígenas, a partir de una experiencia boliviana
}

\author{
GERARDO FERNÁNDEZ JUÁREZ \\ Universidad de Castilla-La Mancha. Toledo
}

RESUMEN

El artículo recoge diferentes sugerencias y reflexiones a partir de una experiencia antropológica de campo realizada en Bolivia sobre las posibilidades que un enfoque intercultural aplicado a la salud puede ofrecer para una mejor relación entre los equipos médicos convencionales y los enfermos indígenas. Las formas indígenas en que se definen la salud y la enfermedad aluden a conceptos cualitativamente muy diferenciados de los correspondientes de la biomedicina. Las medicinas indígenas persiguen, en especial, la recuperación del "ser humano" en su contexto cultural y no tanto del órgano dañado como hace la biomedicina. Aspectos como el género, la concepción del cuerpo, los fluidos o la desnudez, cobran importancia en esas relaciones conflictivas. La experiencia intercultural boliviana permite realizar, finalmente, en el artículo algunas valoraciones últimas sobre inmigración y salud en España.

Palabras clave: Antropología médica, Medicinas indígenas, Interculturalidad, Bolivia.

\section{SUMMARY}

The author offers a number of suggestions and reflections after a fieldwork experience in Bolivia let him explore the merits that an intercultural approach applied to health care-related issues have for a better relationship between westernized physicians and their indigenous patients. Indigenous definitions of health and illness refer to concepts that are qualitatively very different from those used in Bio-medicine. Indigenous drugs are especially intended more for the recovery of the "human being" in his or her cultural context than for the cure of the sick organ on which the bio-medical doctor focuses. Issues such as gender, perception of the body, bodily fluids and nakedness are critical in this potentially conflictive environment. From Bolivia's intercultural experience one can draw some insights into the health care-related problems arising in Spain in connection with the growing immigrant population in the country.

Key words: Medical Anthropology, Indigenous Medicine, Interculturality, Bolivia.

Durante los años 2001-2003 un grupo de antropólogos de la Universidad de Castilla-La Mancha, realizamos una investigación en Bolivia con

RDTP, LX, 2 (2005): 29-53 
la intención de recoger información acerca de las consideraciones indígenas que sobre los conceptos de salud y enfermedad es necesario tener en cuenta para evitar conflictos y malentendidos en la aplicación de políticas interculturales de salud. Es decir, dentro de los intereses de la antropología médica o de la antropología de la salud y de la enfermedad, pretendíamos incidir en la necesidad de afrontar, de una forma decidida y aplicada, un enfoque intercultural de la salud y la enfermedad en contextos indígenas de Bolivia con la esperanza de que este tipo de investigación pudiera aportar datos y sugerencias concretas para el mejor entendimiento entre los diferentes actores implicados en actividades relacionadas con la salud y la enfermedad en dichas poblaciones indígenas. Fruto de las investigaciones realizadas entonces y de los contactos propiciados con otros investigadores sensibles a esta temática ha resultado una publicación colectiva (Fernández coord. 2004). Anteriormente, y gracias a la colaboración establecida en 1995 y 1996 entre el Departamento de Antropología de España y América del CSIC en España y el Centro de Investigación y Promoción del Campesinado de La Paz (CIPCA), llevé a cabo un proyecto postdoctoral en territorio aymara con el Equipo de Salud Altiplano en la zona de Jesús de Machaqa (Provincia Ingavi del Departamento de La Paz). La experiencia de aquellos dos años de investigación se concretó en otra publicación coauspiciada por CIPCA, el Ministerio de Salud y Previsión Social boliviano y la Organización Panamericana de la Salud (OPS) (Fernández 1999). La cooperación italiana viene desempeñando en la ciudad de Potosí una maestría sobre salud e interculturalidad con la participación de profesionales de diferentes sectores sanitarios del Departamento de Potosí, que se pretende posteriormente ampliar a todo el continente americano (Campos y Citarella 2004). Igualmente, la organización yoltpatli, constituida por médicos y antropólogos, algunos de ellos vinculados con la Universidad Nacional Autónoma de México (UNAM), desarrollan diferentes actividades y publicaciones que pretenden sensibilizar y potenciar la aplicación intercultural en el marco de la salud en pueblos indígenas de México (Yolphatli 2003). La Comisión Europea está apoyando en la actualidad un proyecto sobre salud e interculturalidad en la localidad peruana de Ayacucho; del mismo modo, el Programa de Salud de la Agencia Española de Cooperación Internacional está apostando, en cooperación estrecha con el Servicio Departamental de Salud de La Paz (SEDES-La Paz), por el desarrollo de una Diplomatura en Salud Intercultural que se aplicaría en el dominio del departamento paceño en breve.

Todas estas iniciativas no hacen sino incidir en el hecho cualitativamente significativo de que las instituciones y autoridades políticas, académicas y sanitarias empiezan a valorar de una forma positiva las aplicacio- 
nes interculturales en el terreno de la salud y el necesario componente antropológico que deben incorporar para el análisis de la compleja realidad intercultural no sólo de Bolivia, donde registramos la existencia de cerca de cuarenta grupos lingüísticos (Albó 1995), sino de la mayor parte de los Estados de América Latina ${ }^{1}$.

Hace tiempo coincidí en el aeropuerto Internacional de El Alto de La Paz con un ex ministro de educación. La conversación nos llevó hacia la interculturalidad como objeto de reflexión; todavía recuerdo sus palabras. «En Bolivia hoy en día nadie discute la importancia de la interculturalidad y su vigencia, pero, nadie sabe qué es eso, ni cómo aplicarlo". El propio concepto de interculturalidad alude al principio incuestionable de relación entre iguales, donde las relaciones de poder deben encontrarse en un marco adecuado de simetría en que los contendientes muestran una disposición y un talante adecuado hacia el aprendizaje activo y respeto mutuo. Los extremos de corte "fundamentalista" que tratan de desacreditar al otro por sus diferencias estarían fuera de cualquier talante intercultural (Albó $2004)^{2}$.

La influencia del entramado cultural en los distintos escenarios que afectan a la salud y la enfermedad, se acentúan en contextos multiculturales como los presentes en Bolivia, particularmente en el caso de poblaciones indígenas. Los recelos, desconfianzas y dudas que siembran los sistemas médicos entre aquellos potenciales usuarios que no comparten el código de referencia en el que han sido creados histórica y culturalmente, dan lugar a dificultades importantes en la aplicación de dichos sistemas, por muy bondadosas que se supongan sus virtudes. Esta es la situación por lo que respecta a la dinámica de relaciones entre la biomedicina, la medicina "científica", alopática o convencional, como quiera que se prefiera denominar, o el modelo biomédico hegemónico ${ }^{3}$ y los otros siste-

${ }^{1}$ La preocupación académica por la interculturalidad aplicada a la salud, no es nueva, a pesar de haberse puesto "de moda" recientemente de la mano de los procesos migratorios en Europa; recordemos el libro de Aguirre Beltrán (1955) en los años cincuenta del pasado siglo. Resulta paradójico que sea el fenómeno inmigratorio en Europa el dinamizador actual de la interculturalidad, aplicada a la salud, y que no lo haya sido de forma institucional y rotunda, salvo en casos y ejemplos concretos y recientes, la realidad multicultural y sus necesidades de diálogo intercultural en ámbitos dispares de la vida social, cultural y política de América Latina.

${ }^{2}$ Medicina intercultural: "Práctica y proceso relacional que se establece entre el personal de salud y los enfermos, donde ambos pertenecen a culturas diferentes, y donde se requiere de un recíproco entendimiento para que los resultados del contacto (consulta, intervención o consejería) sean satisfactorios para las dos partes" (Campos Navarro 2003).

3 Término que debemos a Eduardo Menéndez (1981; 1992). 
mas incorporados, en el caso de Bolivia, entre otras posibilidades, por las medicinas indígenas de los pueblos originarios.

Las dificultades de relación entre ambos modelos se expresan en situaciones que pueden parecer pueriles en ocasiones, trascendentales en otras, pero que en unos casos y otros conforman los argumentos sutiles que dificultan una adecuada relación entre ambos modelos que garantice la capacidad de elección y de complementación de la "oferta" médica, para mejor satisfacción de las necesidades de salud de los pueblos indígenas de Bolivia

\section{COMUNICACIÓN}

Uno de los factores determinantes que pueden facilitar la aplicación de propuestas interculturales aplicadas a la salud en contextos indígenas es la correcta, permanente y diáfana comunicación y respectivo entendimiento entre todos los actores afectados por el proceso salud-enfermedad-atención. En este sentido, nunca se concederá la suficiente importancia al hecho de que los equipos sanitarios convencionales conozcan de forma adecuada la lengua originaria de la zona en la que estén desempeñando, su labor. Esta circunstancia facilita de manera exponencial el desempeño no sólo de cualquier tipo de campaña de atención sanitaria, intervención, programación preventiva, atención materno infantil....etc., sino, muy especialmente, la paulatina integración del equipo médico en la vida cotidiana de la zona. Este aspecto resulta sumamente importante, hay que tener en cuenta que los médicos originarios forman parte del escenario cultural de los "usuarios" de sus servicios, circunstancia que no ocurre con los equipos médicos convencionales, cuya presencia en ciertos sectores es considerada como algo de uso "excepcional" no incorporado a la realidad cotidiana de los pobladores. Indudablemente el hecho de que alguien del equipo médico conozca la lengua originaria facilitará de una manera clara todo el proceso de consulta, sea ambulatoria o en la propia posta sanitaria, así como las intervenciones que resulten pertinentes. Cuando los miembros del equipo médico convencional desconocen la lengua local, las complicaciones se intensifican en las formas de relación con sus potenciales usuarios, dificultando de una forma clara la comunicación entre ambos y construyendo un proceso de aislamiento que resulta nocivo para la propia estabilidad afectiva del equipo médico, generalmente destinado lejos de sus redes afectivas, familiares y profesionales, en ocasiones bajo la obligación, casi penitenciaria, de un "año de provincias" ${ }^{4}$, resuelto en condiciones precarias.

${ }^{4}$ El "Año de Provincias" constituye un requisito indispensable para los egresados de la carrera de medicina y otras especialidades en ciencias de la salud en diferentes 
En este sentido, una sugerencia a tener en cuenta sería la presencia en los equipos médicos desplazados en los ámbitos rurales, así como en las unidades hospitalarias urbanas, de personas especializadas en la lengua local que puedan facilitar la adecuada relación médico-paciente, difícil de por sí en las situaciones de crisis que supone la presencia de cualquier enfermedad o aflicción. Se dirá que en los hospitales bolivianos o en los servicios de atención rurales siempre hay alguien que conoce la lengua para echar una mano, en concreto la mayor parte del personal subalterno, auxiliares sanitarios y enfermeras; lo que quiero decir es que este rasgo habría que valorarlo y reconocerlo con gratificaciones especiales, como parte del servicio inexcusable de calidad que el Estado debe

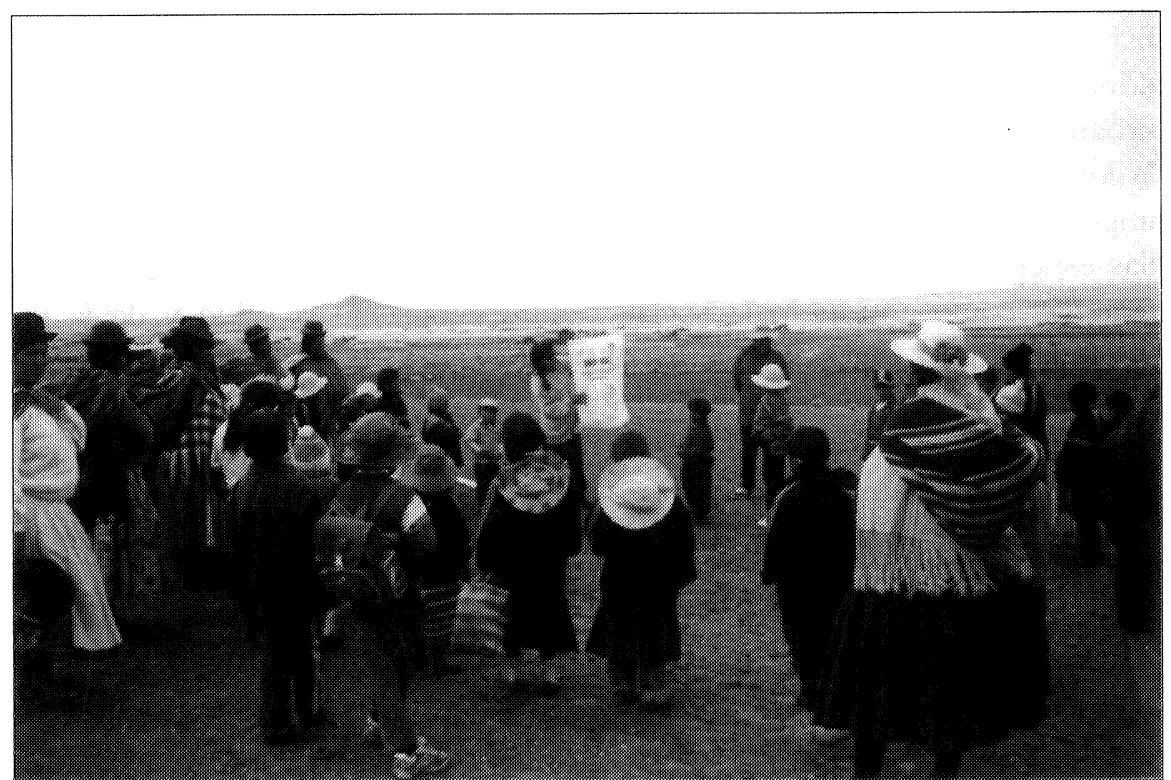

Equipo de Salud Altiplano. Pampas de Machaqa (Bolivia). Campaña de prevención dental (Fotografía: Gerardo Fernández Juárez).

países de América Latina (con diversas denominaciones) por el que se le exige al licenciado la permanencia durante un año, cumpliendo misiones de médico generalista en sectores rurales o de la periferia urbana. En Bolivia este "Año de Provincias" ha sido, de momento, reemplazado por un Servicio Social de Salud sustitutorio que ha rebajado sustancialmente la permanencia de los médicos egresados sobre el terreno, concretamente el "año de provincias" en Bolivia se ha reducido a tres meses, marco temporal que dificulta en forma extrema la integración del médico en el dominio donde pretende realizar su labor asistencial (Flores 2004). El médico anestesiólogo boliviano Dr. Jorge Molina me comentó que según sus datos, contrastados con otros colegas suyos que realizaron el "año de provincias", en el $95 \%$ de los casos, el médico no regresa jamás al lugar donde desempeñó su labor durante el "año de provincias". 
ofrecer a sus ciudadanos para una mejor relación con los servicios de salud de las postas médicas y unidades hospitalarias.

Indudablemente, este aspecto exige valentía y coraje por parte de las autoridades estatales en salud. El cuerpo médico convencional se "defenderá", esgrimiendo que constituye un agravio comparativo para los buenos médicos no hablantes de lenguas originarias y que lo que debe hacerse es premiar a los mejores médicos, hablen o no aymara $u$ otras lenguas originarias; sin embargo será preciso introducir nuevos factores como este lingüístico reconocido pública y políticamente para cambiar y corregir la escenografía tradicional de salud en Bolivia. La dificultad inherente con respecto a este rasgo es que el Estado y la sociedad hegemónica no indígena de Bolivia establezcan la disyuntiva errónea entre médico hablante de lengua originaria y mala calidad, como sucede en el ámbito educativo aplicado especialmente en los dominios rurales frente a los urbanos. Este elemento relativo a la obligatoriedad del conocimiento de la lengua indígena mayoritaria en la zona donde se forma el médico, sería importante introducirlo también en el currículo universitario de todas aquellas especialidades relacionadas con las ciencias de la salud, junto con otras materias como antropología social y antropología de la salud y de la enfermedad, a lo que me referiré posteriormente. En este sentido, habría que afinar mejor en la definición de las prácticas del "año de provincias" para que los médicos licenciados pudieran realizar su labor en el área lingüística y cultural en que han sido formados y no ser enviados a remotos lugares cuya lengua desconocen; demasiada vocación hay que tener para aprender aymara, quechua, y tupí guaraní, además de ser buen profesional de la medicina, evidentemente.

Otro rasgo importante a considerar es que de cara a la eficaz y paulatina aceptación del enfoque intercultural aplicado a la salud sería conveniente, como recoge el Dr. Xavier Albó (Albó 2004), incorporar al sistema médico convencional a los que ya saben la lengua, potenciando la presencia de estudiantes de las diferentes naciones originarias de Bolivia en las distintas carreras universitarias relacionadas con las ciencias de la salud. Es indudable que este sería un recurso muy eficaz para un proceso activo de reconocimiento intercultural aplicado a la salud en todo el Estado.

Si bien el conocimiento de la lengua originaria resulta importante, no es el único factor a tener en cuenta. De hecho, en algunos casos, médicos rurales de indudable adscripción indígena, con un conocimiento claro de la lengua originaria, la emplean siguiendo criterios exclusivamente biomédicos, sin atender a las peculiaridades culturales de su paciente y desconociendo, o no queriendo incidir en ellos por los prejuicios de la 
clase médica occidental, en los posibles síndromes de raigambre cultural que afectan a su paciente o le provocan miedos y desconfianzas con respecto a la actuación del médico convencional.

Cuando nadie del equipo médico conoce la lengua, hay que acudir necesariamente a intérpretes, como he dicho antes, por lo general personal sanitario subalterno de adscripción indígena de los equipos de salud, al que no se premia ni se reconoce en prestigio y méritos por este hecho, como tampoco se hace con los escasos médicos que gozan de esta facultad: el conocimiento de la lengua originaria se supone que "emana" naturalmente de su condición indígena y que es su "obligación" secundar al médico sin más. Vuelvo a repetir que esta cualidad, recomendable en las relaciones médico-paciente en un país como Bolivia, debe ser reconocida y premiada con el puntaje pertinente, a pesar de las presumibles "presiones políticas" en contra de los colegios médicos.

El talante intercultural no depende exclusivamente del buen o mal manejo de las lenguas originarias como factor de comunicación. El grado de empatía que los miembros de los equipos médicos puedan impulsar resulta igualmente importante, casi trascendental, para la incorporación y reconocimiento del equipo médico en el entorno comunitario (Dibbits y De Boer 2002). El deseo y la profesionalidad de un buen médico que se apoya en un intérprete para tranquilizar a su paciente, recabar la información pertinente para un diagnóstico sistemático y formular las pautas para un tratamiento subsecuente, resulta fundamental. En estas circunstancias hay que tener muy presente que el enfermo haya comprendido perfectamente el proceso de su dolencia y el tratamiento a seguir. A veces, el trauma de la consulta médica y el deseo de agradar al médico o la propia culpabilización que en no pocos casos se hace al propio enfermo por su situación, puede agobiar en tal medida a los usuarios indígenas que estén resueltos a escaparse con la mayor rapidez de la consulta (Rance 1999; Dibitts 1994). Los miembros del equipo de salud, bien por su conocimiento de la lengua o mediante intérpretes, deben asegurarse de que el enfermo ha comprendido a la perfección la prescripción médica de que ha sido objeto. Es preciso evitar las confusiones y perífrasis de la jerga médica que redunda en la confusión más absoluta (Fernández 1999). El estilo verbal ha de ser lo más coloquial posible incluyendo frases sencillas y cortas.

La adecuada comunicación entre médico y enfermo precisa además de un conjunto sutil de formas de comunicación no verbales que hay que tener muy en cuenta en contextos indígenas. Si comparamos el proceder del equipo biomédico en la consulta de la posta con la del médico originario en la casa del enfermo, apreciamos de inmediato claras diferen- 
cias en relación con los escenarios de atención terapéutica y las maneras de proceder de ambos. Los sistemas de diagnóstico de la enfermedad y de los cuidados preceptivos son realizados en la casa del enfermo, adonde el médico originario se desplaza para atender, lo que facilita la comodidad del enfermo y su completa confianza, dado el refuerzo emotivo y simbólico que supone contar en todo momento con la compañía de sus familiares. El médico originario está perfectamente imbuido de los caracteres socio-culturales que su presencia en casa del enfermo acredita, cumpliendo de manera exquisita los reglamentos establecidos por la cortesía del grupo étnico de que se trate. En el caso aymara, no se puede entrar en una casa ajena sin advertir de lejos la presencia. Así, una vez concedido el permiso por parte de los titulares de la casa, y tras los consabidos abrazos de rigor como saludo, el yatiri ${ }^{5}$ aymara se ajusta a la perfección a la normativa social establecida, realizando su servicio habitualmente de noche y sabiendo que, en un principio, han de ser los familiares del doliente los que marquen la pauta de actuación que comenzará con el pijchu acostumbrado de coca que propiciará una primera aproximación hacia el problema del enfermo y luego, tras una probable cena, comenzará una actuación terapéutica que le llevará fácilmente toda la noche o varios días con sus noches. Lo que quiero decir es que, desde la perspectiva aymara, el uso del tiempo presenta connotaciones propias muy útiles en la comunicación verbal y no verbal entre los médicos originarios y sus pacientes. En realidad, es considerado de "mala educación" y una descortesía el "ir al grano" en la atención. Todo tiene su adecuado acomodo en el marco de una conversación prolongada que facilita de modo extremo la confianza entre el yatiri o médico originario, que no tiene por qué ser de la propia comunidad, con respecto al enfermo y su entorno familiar. Si comparamos el proceder de los equipos biomédicos en la consulta de la posta, apurados por la prisa, sin los preámbulos necesarios en la adecuada formulación de la cortesía aymara, podemos reconocer una cierta carencia logística importante en la pertinente comunicación médico-enfermo. Las características y conceptualizaciones simbólicas sobre eficacia y eficiencia, aplicadas a la salud son diferentes en ambos modelos. En este sentido, la comunicación entre el médico y el contexto familiar del enfermo, de cuyo perfecto acomodo cabe pensar en la mejor calidad y reconocimiento de la asistencia prestada por los servicios de salud convencionales, depende no sólo del buen conocimiento de las lenguas originarias y de la disposición hacia la empatía y servicio

5 Yatiri. Sabio, especialista ritual aymara, diestro en la consulta de hojas de coca y en el tratamiento de diversas enfermedades de filiación cultural (Fernández 2004). 
profesional en el grupo étnico donde el equipo realiza su labor, sino en la adecuación al código no verbal que el enfermo formula habitualmente en situación de salud.

La comunicación de los pacientes indígenas con respecto a sus médicos originarios constituye el inicio de la fase terapéutica. De hecho, algunas personas se acercan a la posta fundamentalmente para hablar con los médicos o con el personal de salud, los auxiliares aymaras, que mejor se adecuan a sus características culturales. Hablar, conversar es "hacer medicina" desde la perspectiva aymara, cosa que desespera a los equipos convencionales, ya que según nuestros criterios de eficiencia médica este aspecto se considera, por lo común, una pérdida de tiempo. Localizar la causa de la enfermedad, saber lo que a uno le pasa, superar la angustia e incertidumbre, contiene en sí mismo un principio terapéutico importante que hace que, en ocasiones, una vez que el enfermo y su familia saben lo que le pasa, suspenden el tratamiento biomédico, puesto que el conocer la causa de la aflicción o patología constituye, en sí mismo, un referente terapéutico primordial ${ }^{6}$. En este caso, el equipo biomédico debería conocer la importancia de la comunicación verbal y no verbal en el trato con sus enfermos originarios y hacer gala de la misma, en la medida de sus posibilidades reales, toda vez, repito, que la "charla" facilita la relación y confianza con el enfermo y forma parte de su propio proceso terapéutico.

\section{EXAMEN Y ATENCIONES MÉDICAS: LAS ACTUACIONES}

Las formas de examen del enfermo deben ser consensuadas y nunca impuestas; es decir, hay que indicar poco a poco lo que se va a realizar con permiso del paciente y el efecto que puede suponer sobre su cuerpo. Así, por ejemplo, cuando sea preciso utilizar el fonendoscopio hay que indicar para qué se hace y por qué es necesario descubrir la parte del cuerpo correspondiente, así como advertir de la sensación de "frío" que produce; hay que tener en cuenta que algunas de las enfermedades indígenas están conceptualizadas en torno a los valores simbólicos de lo "frío" y "ć́lido" y, quizá, el efecto de la consulta médica puede resultar

\footnotetext{
${ }^{6}$ Por esta circunstancia, algunos médicos me han confesado su desesperación al comprobar que los indígenas no continúan con el procedimiento terapéutico hospitalario en el caso de las placas de rayos $\mathrm{x}$; una vez que tienen las radiografías en las que se puede "ver" la causa del mal se dan por contentos y no continúan el procedimiento. Este tipo de "deserción" hospitalaria del paciente tiene en cuenta otros aspectos, entre ellos el económico, que veremos luego.
} 
pernicioso al alterar este equilibrio, no sólo térmico, sino muy especialmente simbólico ${ }^{7}$. La desnudez del cuerpo, sobre todo de las mujeres, en la atención médica convencional choca violentamente con las categorías y conceptualizaciones indígenas, particularmente en los Andes; por ello es preciso atender y examinar en lo posible bajo la ropa ${ }^{8}$.

Hay que tener en cuenta las dificultades existentes de acceso al agua en muchas áreas rurales, particularmente del altiplano; esto hace que el agua sea algo que se emplee fundamentalmente para beber y cocinar y para atender al ganado y sólo después, y en un apartado lugar, para el aseo personal. La inexistencia habitual de agua corriente en los hogares hace del agua un bien escaso y costoso, ya que hay que recorrer grandes distancias para conseguirlo. En estas condiciones la higiene diaria es un lujo gratuito y en ocasiones poco apetecible, dadas las características de frío e intensas heladas del invierno altiplánico?. Un médico con formación curricular relativa al marco cultural donde realiza su servicio, valorará las dificultades de acceso al agua y por tanto a la higiene corporal, evitando grotescas apreciaciones sobre la suciedad de los enfermos indígenas que le visitan en la posta (Fernández 1999).

\subsection{Sangre y Orina}

En ocasiones, el examen médico convencional prescribe análisis de sangre y orina. La concepción aymara sobre los fluidos orgánicos resulta particularmente importante, al igual que sucede en otros grupos amerindios. La sangre es un bien limitado, no se reproduce con facilidad; es como si naciéramos con la cantidad de sangre que debemos emplear toda la vida. Por otro lado, los aymaras insisten en que su sangre cuesta cara y no puede derramarse ni extraerse de forma arbitraria. Los derramamientos de

\footnotetext{
${ }^{7}$ El complejo cultural sobre lo "cálido" y lo "fresco" está presente, dentro de los síndromes de filiación cultural, en la mayor parte de los pueblos amerindios y resulta suficientemente conocido. En el dominio aymara estas categorías simbólicas están presentes en diferentes ámbitos como los procesos orgánicos fríos y cálidos; los alimentos fríos y cálidos; productos de la farmacopea tradicional fríos y cálidos e incluso el propio talante de los seres humanos igualmente "fríos" o "cálidos", exigiendo un tratamiento personalizado para las enfermedades ya sean cálidas o frescas (Foster 1980; Gutiérrez 2000).

${ }^{8}$ Me comentaba una médico de Potosí: "...jcómo una mujer que no se desnuda para hacer el amor con su compañero va a hacerlo para la revisión del médico a quien no conoce!n

${ }^{9}$ De hecho, la repulsión por el baño se inicia en edades tempranas donde se amenaza al niño que comete actos inapropiados con un baño frío.
} 
sangre deben ser indemnizados por la pérdida ocasionada, concebida como irreparable ${ }^{10}$. Es necesario explicar de forma sencilla las razones y uso del análisis sanguíneo como medio, a la manera de las hojas de coca, para ver la enfermedad ${ }^{11}$, a la vez que aliviamos las desconfianzas sobre el uso que el médico hace de la sangre extraída. En el altiplano, al menos, es muy frecuente el abuso latente que los naturales suponen a la tendencia a la extracción sanguínea por parte del médico ${ }^{12}$, así como la asimilación del médico con el "Operador" ${ }^{13}$, el temible kharisiri. El kharisiri, antaño relacionado con la iglesia colonial en esas relaciones de poder completamente asimétricas, salpica en la actualidad a diferentes colectivos, entre ellos al sanitario. No en vano el kharisiri recorre las comunidades aymaras, especialmente en el mes de agosto, recolectando grasa y sangre con su ajeringa" para, "pagar la deuda externa boliviana". Un equipo médico asociado simbólicamente en sus actuaciones al perfil del kharisiri está materialmente condenado en al altiplano al más atroz de los aislamientos. Lógicamente, si esto se aplica a los análisis sanguíneos, con mayor importancia a las operaciones quirúrgicas.

Con respecto a la orina, ésta se emplea habitualmente en los tratamientos médicos indígenas en diferentes casos (Absi 2005) e incluso en los procedimientos de diagnóstico terapéutico ${ }^{14}$. El consumo de orina y

${ }^{10}$ En las borracheras siempre que se produce algún enfrentamiento con derramamiento de sangre, la víctima afectada por la pérdida y su familia suelen realizar una solicitud al médico para que evalúe y certifique la cantidad de sangre derramada, para realizar la denuncia y solicitud correspondiente de indemnización económica al agresor y su familia. Recuerdo el caso de Manuel Coa , residente aymara en la barriada alteña de Villa Huayna Potosí cuando, afectado por una úlcera sangrante y tras un vómito de sangre importante, increpaba asustado al equipo médico que pretendía sacarle sangre para su análisis ... “ino tengo más sangre!"

${ }^{11}$ Los yatiris aymaras emplean diferentes sistemas para el diagnóstico médico, como son las hojas de coca, la orina del enfermo, o la disección de animales que previamente se han pasado por la zona afectada del cuerpo del enfermo produciendo lo que se conoce como "limpia" ritual (Fernández 2002; 2004).

${ }^{12} \mathrm{El}$ temor a las extracciones sanguíneas se ve "confirmado" por las disposiciones de los equipos médicos en las instituciones hospitalarias, en relación con los grupos sanguíneos y las necesidades de los enfermos ".... a otros coloca, ¿no ve?"; "para vender a otros saben sacar".

13 "Operador" denominan en castellano al kharisiri en el Cantón de Ajllata. Indudablemente este calificativo dificulta nuevamente el reconocimiento del médico como alguien competente en sanar y lo acerca a la figura perversa del personaje mítico (Fernández 2004b).

${ }^{14}$ Las formas que adquiere la espuma de la orina del enfermo y sus características sensibles son analizadas por los yatiris aymaras en el diagnóstico de enfermedades. 
su carácter "cálido" le capacita en el tratamiento de ciertas enfermedades indígenas, así como por sus propiedades como desinfectante y antihemorrágico.

\subsection{Cirugía}

La operación quirúrgica supone un trauma cultural, económico y social para los afectados indígenas. Cultural por las connotaciones negativas que implica la manipulación interna del cuerpo, donde nadie tiene que "mirar"; económico por el monto, inalcanzable para la gente del campo, de las cifras que habitualmente se manejan en cualquier operación quirúrgica, con el añadido del desplazamiento al hospital donde vaya a realizarse la intervención; social por la toma de decisiones familiares, domésticas e incluso comunitarias que implica el traslado hospitalario y el temor que produce enfrentarse a un espacio frío deshumanizado sin previsión espacial para acoger a los familiares que acompañarán, con seguridad, al enfermo ${ }^{15}$. Lo ideal sería dotar a las postas sanitarias con personal perfectamente capacitado, en formación específica y medios técnicos, para poder realizar in situ, es decir, en el campo, las intervenciones quirúrgicas de corte sencillo, para evitar en lo posible el traumático traslado de los pacientes al hospital; circunstancia que en ocasiones supone la negación absoluta no sólo de la familia del afectado sino de la propia comunidad, con sus autoridades al frente, a las recomendaciones del equipo de salud.

Por otra parte, los grupos indígenas del altiplano recelan de las prácticas quirúrgicas debido al reconocimiento ético y moral que posee el cuerpo completo. Los difuntos deben enterrarse con todos sus restos orgánicos y corporales ${ }^{16}$. El costo de las intervenciones quirúrgicas y el

${ }^{15}$ La Confederación Sindical Única de Trabajadores Campesinos de Bolivia (CSUTCB) paralizó la ley de trasplantes de órganos por la reticencia, desconfianza y abuso que se supone realizan en las instituciones hospitalarias, concepto asociado al tráfico de órganos y la idea indígena del "cuerpo completo", incompatible con esta norma, que comento más tarde.

${ }^{16}$ Esta valoración ética indígena sobre el cuerpo completo dificulta en gran medida las prácticas de la cirujía convencional, así como la puesta en práctica de un sistema nacional de transplantes de órganos, como expuse arriba. He recogido en el Lago Titicaca varios casos de personas abocadas a la amputación de alguna de sus extremidades y miembros que han saltado literalmente de la camilla hospitalaria para curarse en sus comunidades de origen con los médicos aymaras mediante cataplasmas realizadas con diferentes especies herbáceas de la zona y barros de calidades de tierras distintas, que han sido suficientes para detener aparentes procesos de gangre- 
maltrato que se espera en las unidades hospitalarias, constituyen serios rechazos para las intervenciones quirúrgicas. Los indígenas acusan al médico de que está siempre preparado para la "operación", por su avidez para curiosear las interioridades humanas y por su deseo de riquezas. Se sospecha que la cirugía responde a los intereses profesionales y económicos del médico y no tanto a las necesidades terapéuticas del paciente.

\subsection{Parto}

El parto incorpora otra serie de características culturales peculiares de las poblaciones indígenas que chocan con la pragmática valoración y medicalización del alumbramiento desde la perspectiva biomédica ${ }^{17}$. La posición de la mujer durante el parto, para buena parte de los grupos amerindios, establece disposiciones muy diferentes a las del modelo biomédico. La posición más adecuada es en cuclillas, en ocasiones con la asistencia del marido por detrás, masajeando el abdomen de la parturienta bajo la atenta mirada de la partera, en contacto con la tierra, con la enorme carga simbólica que este contacto tiene, particularmente en los Andes. En las comunidades aymaras es preciso entregar la placenta a la madre, ya que desde su perspectiva es el "hermano" del neonato, cuya vida no va a prosperar. La madre da a luz al niño y a su placenta, algo así como el "hermano monstruoso"; en el altiplano aymara hay que compensar al menos afortunado, en este caso a la placenta que no va a prosperar vitalmente, frente a la criatura recién nacida ${ }^{18}$. Por ello, las familias adornan la placenta con los rasgos característicos del nacido; si es niña, se adorna su placenta con mixtura y con elementos en miniatura que

na. Hay que destacar en este mismo sentido, la solicitud ocasional, por parte de los familiares del paciente intervenido quirúrgicamente, de los algodones impregnados con su sangre (Óscar Velasco, comentario personal) o la frecuente solicitud de la placenta tras el parto, como veremos luego. Incluso los cabellos cortados de las personas son objeto de custodia y cuidado (Fernández 2002).

${ }^{17}$ Algunas enfermeras destinadas en las unidades maternoinfantiles de hospitales en España, sensibilizadas con la antropología, me han confirmado que los equipos de intervención en el parto se refieren con frecuencia al neonato como "producto", lo que asemeja su actuación a la de una especie de "factoría industrial. Muy diferente es la perspectiva desde las consideraciones emic de las poblaciones indígenas, donde existe un conjunto de categorías de significación cultural y cognitivas muy representativas que afectan a todo el proceso del parto configurando incluso una terminología específica (Dibbits y Terrazas 2003; Arnold y Yapita 1999).

${ }^{18} \mathrm{El}$ propio feto humano es objeto de consideraciones culturales que nos ofrecen información precisa sobre algunos comportamientos indígenas (Platt 2001). 
reflejan la variable de género del nacido, en caso de niña, se enterrará acompañada de sus polleritas, su pequeña cocinita (qbiri awichu)...etc, en caso de niño, con una yunta en miniatura....etc. Cualquier actuación arrogante sobre la "ignorancia" de las poblaciones aymaras por este rasgo cultural, o manifestaciones prepotentes del equipo médico sobre la inoportunidad e inconveniencia de esta manifestación cultural redundará con certeza en el fracaso, primero con respecto al deseo de que las parturientas acudan a la posta médica, y luego en la conveniente articulación del equipo médico en el seno de la comunidad. Una parte del considerable rechazo de las parturientas a acudir a las postas médicas para dar a luz se debe a la importancia simbólica de hacerlo en casa y en contacto con la tierra; dar a luz fuera de casa, del calor del hogar, según las prescripciones del binomio "cálido-fresco" con que algunos procesos orgánicos, como el parto, se ven afectados, supone "parir extraños", es decir, fuera del linaje doméstico.

Por otro lado, el proceso del alumbramiento es un proceso "cálido", la parturienta indígena en el altiplano aparece con gran cantidad de ropa, secundada por la aysiri, la mujer partera, para provocarle la sudoración correspondiente del proceso "caliente" que supone el dar a luz. Uno de los motivos frecuentes de rechazo de las mujeres aymaras en relación con el parto hospitalario se debe al frío de los hospitales, con sus grandes salas y sus protocolos de actuación en los que la mujer parturienta aparece ligera de ropa. Esta situación incomoda en gran medida a las mujeres indígenas provocándoles no pocos recelos e incluso patologías iatrogénicas por el contraste entre su modelo cultural relacionado con el parto y lo que se encuentran en el parto hospitalario protocolizado.

\subsection{Vacunación y fármacos}

La medicina preventiva, en lo que respecta a las campañas de vacunación, debe partir de una información previa, concisa y clara sobre lo que se pretende y los beneficios que el proceso de vacunación va a suponer para la población local. Es preciso proyectar unos días o semanas antes una exhaustiva campaña de información previa, in situ con las autoridades comunitarias y el resto de los pobladores interesados en la información. No puede esperarse que, en el crítico momento, la gente de la comunidad, sin previo aviso de lo que se pretende, acepte complacida la llegada del equipo de salud y mucho menos que se preste a donar a sus hijos para que les claven agujas. Existen categorías y conceptos de enfermedad en las diferentes poblaciones amerindias, particularmente en las tierras bajas del bosque amazónico, según las cuales la enfermedad 
se debe a algo que se nos introduce en el cuerpo ${ }^{19}$. Igualmente hay recelo sobre el objetivo "real" de las vacunas, dada la desconfianza que las poblaciones indígenas tienen sobre cualquier propuesta o iniciativa externa que escape de sus parámetros de control; así se dice que las vacunas pretenden esterilizar a las mujeres indígenas, o hacerlos sumisos y sin voluntad contestataria frente a los intereses del Estado ${ }^{20}$. Por otro lado, el rechazo a las campañas de vacunación presenta otras consideraciones de características simbólicas; recordemos el complejo ceremonial del kharisiri con su "jeringa" recolectora de grasa y sangre, así como la certeza de que el uso de los medicamentos acomodan el cuerpo de los originarios, haciéndolos más vulnerables y dependientes de la biomedicina, a la vez que los aleja de los remedios tradicionales.

Con respecto al uso de los medicamentos occidentales hay que insistir en que igualmente tienen su sentido y valor desde la perspectiva indígena. Los sabios aymaras (yatiris) por ejemplo, diferencian entre las "enfermedades de la gente" y las "enfermedades del doctor", delegando en uno u otro según su saber hacer. Las enfermedades del doctor aparecen reconocidas bajo los términos castellanos "inyección", "operación", "transfusión" que muestran, desde la perspectiva indígena, lo más violento de una biomedicina centrada en el cuerpo orgánico, mientras que la medicina aymara, como la de otros grupos amerindios, centra sus necesidades terapéuticas en el ser humano, utilizando todos los recursos que incorpora, en cada caso, el concepto de persona. En este sentido, no resulta infrecuente que en ciertas ocasiones los originarios busquen al médico "de mandil blanco" para utilizar sus herramientas terapéuticas, una vez identificado que el caso "es para el doctor". Sería conveniente conseguir, una vez sensibilizado el sector biomédico a través del pensum universitario, la capacidad que igualmente debieran tener los biomédicos de delegar, más allá de los compromisos de totalidad adquiridos por el ajuramento hipocrático", en el médico originario en el caso de las "enfermedades de la gente", que no está capacitado para atender correctamente.

Los medicamentos empleados en la posta médica no deben ser regalados o gratuitos, ya que se considera que lo que se da gratis no es eficaz o está caducado o bien pretende otros fines encubiertos, dañinos para la población. Los medicamentos y "tratamientos" que cuestan dinero, son

\footnotetext{
${ }^{19}$ Entre los Yagua amazónicos del Perú, existe todo un componente etnovírico sobre dardos chamánicos que se introducen en el cuerpo del afectado provocándole enfermedades (Chaumeil 2004).

${ }^{20}$ Este tipo de discurso resulta muy frecuente entre los representantes del magisterio rural aymara.
} 
considerados "poderosos" y eficaces por los usuarios indígenas de los servicios de salud. De igual manera, entre los aymaras, las atenciones curativas rituales y las ofrendas ceremoniales costosas se consideran más eficaces, con la capacidad de incidir de forma proyectiva sobre el cuerpo del doliente sin necesidad de abrirlo, como hace el cirujano. Obviamente, esta cualidad relativa al coste de los medicamentos debe ser empleada con flexibilidad por parte del equipo de salud, según las posibilidades económicas de los pacientes que acuden a la posta, de tal forma que nadie se quede sin su tratamiento completo por razones económicas. En este sentido hay que resaltar los problemas que está provocando en las postas rurales la aplicación protocolaria e inflexible del SUMI (Seguro Universal Materno Infantil), quejándose los médicos rurales de que no pueden recetar fármacos, fuera de las ocasiones y tipos reflejados en los protocolos del SUMI, lo que merma considerablemente su capacidad de decisión y criterio.

La actuación del equipo biomédico en el área rural no puede circunscribirse exclusivamente a la posta médica. Es preciso mantener una comunicación fluida con el entorno comunitario, autoridades originarias, familias, asociaciones del lugar, magisterio rural y por supuesto con los médicos originarios. Resulta imprescindible concretar con ellos de forma clara que no existe ningún deseo de "competencia" y sí de colaboración; la competencia o no de unos u otros, en cada caso, responde a la elección que el propio enfermo realice libre y democráticamente según su criterio.

\section{EFICACIA SIMBÓLICA}

Estar sano o enfermo en las sociedades humanas no depende sólo de la estructura celular y de los componentes orgánicos del cuerpo, sino que cada cultura decide dar sentido a lo que entiende por salud y enfermedad. En poblaciones amerindias de Bolivia existe un complejo conjunto de formulaciones culturales sobre enfermedades, los llamados "síndromes culturales", que recogen aspectos diferenciados de su realidad cultural. Así las cosas, determinadas patologías o aflicciones son resueltas mediante la consideración que se hace del ser humano y sus expectativas funcionales. No tiene sentido la parcelación orgánica, mirar "adentro" del cuerpo o preocuparse por un órgano específico. Los rituales terapéuticos empleados por diferentes grupos étnicos de Bolivia hacen alusión a modelos simbólicos de percepción y concepción del cuerpo y del ser humano en situación de eficacia social, junto con su estructura social y de parentesco, así como a las diferentes cosmovisiones que, según cada caso, se ven afectadas. Por 
eso buena parte de los procedimientos terapéuticos son de carácter ritual y simbólico, de forma paralela al conocimiento prolijo de especies naturales que emplean en su abundante farmacopea tradicional.

Estas soluciones rituales que emplean las poblaciones amerindias en tratar sus problemas de salud chocan con la mentalidad cartesiana y racionalista de los equipos médicos de la posta médica o institución hospitalaria al uso. Aludir a las prácticas terapéuticas del médico originario con respeto y aprecio, recomendando la consulta característica al médico originario y su parecer, cuando la ocasión lo precise, facilitaría en gran medida la comunicación e integración del equipo médico en el área, además de redundar en el propio prestigio y apego de los usuarios indígenas de los servicios de salud. En numerosas ocasiones asistimos a la presencia, casi clandestina, de los yatiris y médicos indígenas originarios en las unidades hospitalarias, a solicitud de los propios enfermos, aprovechando la ausencia momentánea de los equipos médicos, el relevo en las guardias o el turno de noche, lo cual no hace sino incidir de una forma manifiesta en la diferenciación de sentido que con respecto a la propia enfermedad poseen los agentes de salud convencionales y los usuarios indígenas de dichos servicios ${ }^{21}$.

\section{LOS ESCENARIOS}

El hecho de que los centros de salud convencionales, instituciones hospitalarias y postas médicas rurales no sean reconocidas por los posibles usuarios indígenas de dichos servicios como competentes en el tratamiento de la enfermedad, según las concepciones locales, hace que estas instituciones aparezcan mermadas en sus capacidades e infrautilizadas. "El hospital es el lugar donde la gente va a morir" es una frase lapidaria, pero recurrente en el dominio rural de Bolivia, que desacredita las posibilidades en atención médica de las unidades hospitalarias ${ }^{22}$. Sería preciso averiguar cuáles son las razones de este rechazo; cuál es el tipo de relación existente entre el personal médico y sanitario del hospital o de la posta y los respectivos usuarios indígenas de la zona; acreditar el nivel de comunicación habitual entre los agentes de salud del hospital y los usuarios de los servicios; comprobar el grado de satisfacción de los usua-

\footnotetext{
${ }^{21}$ Merece la pena recordar el ejemplo que recoje Larry Dossey (1994).

${ }^{22}$ Hay que tener particular cuidado en la elección del lugar donde se construirá el hospital; con demasiada frecuencia, en el altiplano, los hospitales se erigen cerca de los cementerios indígenas, lo que contribuye a incrementar el rasgo "tabú" de la institución hospitalaria (Jose Luis Baixeras, comentario personal).
} 
rios que acuden a la posta o a la unidad hospitalaria y conocer el fundamento de su rechazo.

La presencia de médicos originarios, yatiris, kallawayas y otros varios posibles en el mismo espacio terapéutico del hospital donde trabaja el médico presenta dificultades importantes. Indudablemente, el hecho de que médico y médico originario compartan espacios en el "centro de poder", tanto de la posta, como del hospital, adquiere connotaciones políticas razonables para la acreditación de un enfoque intercultural aplicado a la salud, pero la cuestión no sólo radica en colocar a uno u otro especialista al lado, codo con codo. Si bien el "escaparate" político de la interculturalidad goza con este tipo de experiencias, es preciso valorar algunos aspectos. En primer lugar la difícil aceptación del propio espacio hospitalario por parte de grupos humanos que no poseen en su bagaje cultural nada parecido a una "casa de curas", ya que es el propio médico originario quien practica la medicina de forma itinerante atendiendo a los enfermos, bien en su propia casa o en la de aquél. Por tanto, difícilmente nadie acudirá a una institución "fría" donde no entienden la lengua originaria ni las consideraciones culturales sobre salud y enfermedad, aquellas consideradas por los lugareños como "verdaderas razones", para

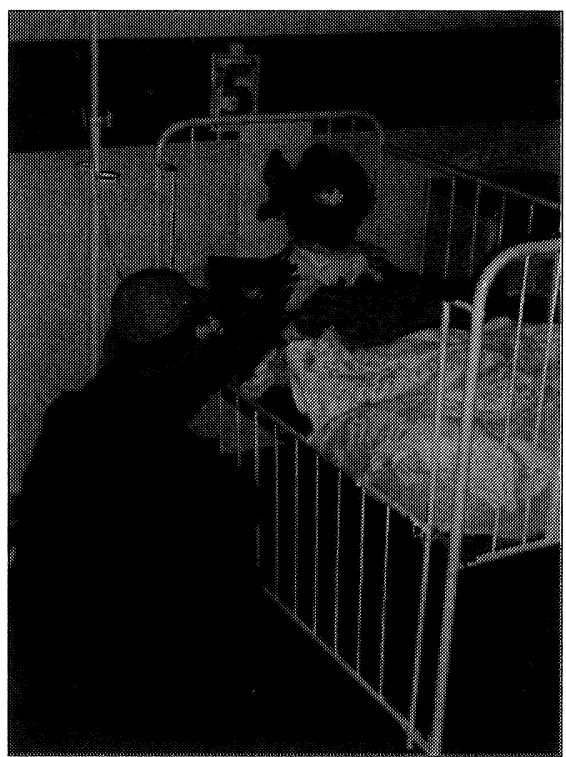

Unidad de pedriatría del Hospital Daniel Bracmonte, Potosí (Bolivia).

Fotografía: Programa de Formación en Salud Intercultural "Willaqkuna". afrontar las crisis y traumas que la enfermedad ocasiona. Si los médicos y personal de salud no están formados en criterios interculturales, difícilmente valorarán las competencias que el médico originario desempeñe en la posta o en la unidad hospitalaria, considerándole, dentro del más aberrante paternalismo, como un pequeño auxiliar de primeros auxilios, para que "aprenda" las verdaderas enfermedades y sepa derivar al hospital los casos importantes. Este tipo de actitud se acerca al sentido de la expresión emplear la de ellos para salirnos con la nuestra", que escuché de un médico rural en su año de provincias, y no ayuda al desarrollo de criterio intercultural alguno, por más que médico y yatiri encuentren acomodo en el mismo escenario terapéutico del 
hospital o de la posta médica. Si el médico originario no deriva los pacientes que no puede atender al biomédico y este a su vez reconoce sus limitaciones en el competente tratamiento de enfermedades que sí puede tratar el médico originario y lo deriva a este, nada se adelanta con hacer compartir a uno y otro especialista el espacio hospitalario ${ }^{23}$.

Otro rasgo a tener en cuenta es que el médico originario de la posta o de la institución hospitalaria debe estar incluido en nómina, es decir, debe recibir su dinero por el trabajo realizado (Flores 2004). Existe la idea de que los médicos originarios reciben la voluntad de sus pacientes y así resulta en el campo, en las comunidades desperdigadas por el altiplano, la selva o el chaco, pero si queremos que sean reconocidos institucionalmente deben ser premiados por su valioso quehacer con su pacientes con el aporte económico adecuado; de otra forma no les compensará realizar su trabajo en un lugar fijo y preferirán continuar con su tradición deambulatoria ${ }^{24}$

Hay que valorar, por otro lado, la reacción de los usuarios potenciales de los servicios: así como en la ciudad de La Paz y otros sectores ya hay dispensarios que ocupan médicos y kallawayas u otros médicos originarios con relativa aceptación, habrá que ver lo que sucede en el dominio rural, con sus características diferenciadas. El médico tiene su aespacio de poder " con las atribuciones que le confiere el mismo y, a su vez, el yatiri posee los suyos y no es bueno mezclar ambos conceptos, porque los pobladores indígenas y los propios médicos originarios recelan de la ineficacia de esa mezcla. Varios yatiris consultados directamente se negarían a trabajar en la posta sanitaria porque no es su ámbito de "poder"; en el caso del yatiri aymara, el escenario que ejerce como caja de resonancia de su poder terapéutico y ceremonial es el propio altiplano, donde se encuentran todos los seres tutelares que avalan su proceder, espacio sagrado que es capaz de reproducir en el dominio doméstico, ya sea en su casa o en la casa del paciente, a través de sus remedios terapéuticos y sistemas de diagnóstico (hojas de coca, mesas rituales...etc.). Indudablemente, el maltrato a los yatiris o la competencia que los médicos puedan tratar de realizar con ellos en nada ayuda a sus disposicio-

${ }^{23} \mathrm{La}$ interculturalidad aplicada a la salud no consiste sólo en introducir médicos indígenas en las postas e instituciones hospitalarias de cualquier forma. Precisa una reflexión global sobre los sistemas de salud y sus características en América Latina .

${ }^{24}$ Tampoco se pueden reglamentar económicamente las actuaciones de los médicos indígenas a imitación de la creación de tarifas por servicios prestados como se hace en la biomedicina; mucho menos pretender que sea la biomedicina quien "a su juicio" estipule y controle el quehacer de los médicos nativos y los "precios" que debe cobrar, como se intentó hacer en Bolivia sin éxito, lógicamente (Flores 2004). 
nes interculturales. Un yatiri me comentó en una ocasión que él nunca trabajaría con el médico en la posta si no era a partir del respeto mutuo y el compartir los procedimientos terapéuticos; se dice que el médico ajuega" con sus aparatos y mira frascos recetando remedios, pero sin comunicar nada, "en secreto" ${ }^{25}$.

Dentro de la propia estructura hospitalaria hay que tener en cuenta los cuidados relativos a los pacientes indígenas ingresados en la institución, como puede ser el incluir en la dieta de los enfermos, en lo posible, los productos alimenticios de la zona; la explicación directa y franca sobre el consumo de fármacos; explicar rasgos sensibles en los tratamientos que se apliquen como sabores, olores, sensaciones orgánicas, cambios térmicos, etc., todos ellos rasgos que afectan a los criterios indígenas sobre salud y enfermedad. Es imprescindible habilitar espacios confortables para los familiares y acompañantes, que serán los que sirvan como "colchón" amortiguador de los temores de los pacientes ingresados. El disponer de espacios habilitados para los familiares y acompañantes de los enfermos redundará en una mayor valoración positiva sobre la posta o institución hospitalaria, con el permiso obvio de visitar al familiar ingresado el tiempo que fuera preciso.

Otro de los rasgos que debe ser norma en la consulta ambulatoria o en la atención hospitalaria es el "secreto"; es decir, parte de los temores y recelos de los indígenas con respecto al hospital y las actuaciones médicas radican en su carácter público. La situación médica de cada enfermo en la relación que establece con el médico indígena sólo le interesa a el propio afectado y su familia; todo debe quedar en este entorno privado, intimista y particular, por el contrario el hospital, con su masificación asistencial y sus colas de enfermos y familiares, va en contra del valor que el dominio indígena otorga a la privacidad de la situación médica. De hecho, en el altiplano aymara, los yatiris, al acudir a la casa del enfermo lo hacen de noche y con la máxima discreción, para que nadie del entorno, ajeno a la familia, pueda advertir su presencia en la casa.

La labor de sensibilización para la aplicación de un enfoque intercultural a la salud debe producirse a la mayor brevedad posible en el pensum de los curricula de todas las carreras universitarias de ciencias de la salud,

\footnotetext{
${ }^{25}$ De hecho, este "secretismo" aparente del médico, debido en gran medida a sus problemas de expresión en las lenguas originarias, va en contra de la aceptación incluso de los talleres organizados por los equipos de salud en la comunidades indígenas; los propios "beneficiarios" indígenas creen que el médico organiza los talleres para "aprender" las técnicas indígenas y no tanto para informar o enseñar las suyas, con lo que algunos prefieren no acudir a los talleres formativos organizados por el equipo de salud.
} 
a nivel del Estado; es la única forma de cambiar el escenario en el que otra forma de pensar la salud sea posible en Bolivia. Junto con la renovación de los curricula de las carreras universitarias de ciencias de la salud es preciso intensificar la sensibilización de los respectivos colegios médicos; premiar en los concursos de plaza e items a los médicos y otros especialistas con conocimiento de lenguas originarias y patrones culturales sobre salud y enfermedad; fortalecer la figura de médicos interculturales con un boleto económico de reconocimiento; dignificar el antiguo "año de provincias" hoy convertido en "Servicio Social de Salud" que, si bien ha conseguido consolidar los items de salud en el dominio rural, supone una experiencia de campo insuficiente para los implicados, con el actual recorte a tres meses en área rural y seis meses en área urbana.

\section{MIRANDO LA INMIGRACIÓN EN ESPAÑA}

Las situaciones que aquí hemos observado sobre las dificultades existentes en la relación entre la biomedicina y los conceptos indígenas sobre salud y enfermedad en un contexto delimitado, como es el caso del altiplano aymara con extensión hacia otras partes de América Latina ipueden sugerirnos algunas ideas y ciertos análisis sobre el proceso migratorio y la salud en España? Se trata de contextos y situaciones diferentes que en algunos casos no admiten comparación posible, pero tal vez merezca la pena entretenernos un poco en la cuestión.

Partamos del hecho significativo de que todos somos de alguna manera "inmigrantes", o nos sentimos en cierta forma como tales, dentro de una institución hospitalaria, con sus ajenos protocolos, su pesada administración o sus códigos de significación peculiares en torno a los colores ${ }^{26}$, los espacios, la comunicación verbal (jerga médica) y no verbal, o los emblemas de poder ${ }^{27}$. Todo estos rasgos configuran una "cultura sanitaria" en el interior del hospital donde el enfermo adquiere un rol con su ingreso al que debe, muy a su pesar, como el inmigrante en territorio "de acogida", ajustar lo antes posible sus normas de comportamiento.

Los inmigrantes llegan con todo su bagaje cultural, con sus preconcepciones de origen sobre salud y enfermedad, y con el modelo, más o menos

${ }^{26}$ En más de una ocasión he preguntado sobre la situación de un familiar al celador de urgencias, creyendo hablar con el "cirujano jefe", debido a los códigos uniformados con sus respectivos colores.

${ }^{27}$ Nada más característico al respecto que el célebre fonendoscopio al cuello y su empleo como emblema identitario de la clase médica, aunque sea en la cafetería del hospital. 
claro, sobre las estrategias de acceso a la biomedicina en su país de origen. Las formalidades para el acceso hospitalario no tienen por qué ser las mismas en el país de origen que en el de "acogida", ni por supuesto se comparten idénticas expectativas con respecto al proceso enfermedadsalud-atención. Por eso, reflexionar sobre cuestiones básicas en la relación médico paciente en contextos interculturales, como el papel fundamental atribuido a la lengua ${ }^{28}$, las concepciones sobre el cuerpo y el concepto de persona, las relaciones de género ${ }^{29}$, las atenciones médico "paciente" ${ }^{30}$, el mobiliario ${ }^{31}$, la alimentación ${ }^{32}$, los fluidos corporales ${ }^{33}$ y otros aspectos semejantes, forman parte del patrimonio cultural que los inmigrantes traen consigo y emplean, como lo hacemos nosotros, al hacer comprensible la situación anómala que la enfermedad y sus aflicciones causan en nuestras vidas (SEMFYC 2004).

Si bien es cierto que las peculiaridades culturales funcionan en la forma diferenciada como los seres humanos damos sentido al proceso enfermedad-salud-atención, es preciso actuar con suma sutileza y cuidado para que estas "diferencias" no se constituyan en meras referencias exóticas,

${ }^{28}$ ¿Por qué no incorporar en nómina por parte de las instituciones hospitalarias a personas bilingües que se expresen perfectamente, además de en castellano, en la lengua más representativa del colectivo de inmigrantes con mayor presencia en la zona? Recuerdo el caso que me comentó la $\mathrm{Dr}^{\mathrm{a}}$ Luisa Abad, miembro de nuestro equipo de investigación, cuando realizaba sus prácticas asistenciales en el complejo hospitalario de La Paz en Madrid. Se encontró "aparcado" en una silla de ruedas a un viejito peruano que se expresaba en quechua y que sus compañeras de turno habían desviado hacia el servicio de psiquiatría por presuponer que la naturaleza de dichos "Sonidos" sólo podía deberse a un proceso de demencia senil.

${ }^{29} \mathrm{La}$ presencia de los miembros de la familia, particularmente mujeres, en la atención de los partos y su implicación en el acompañamiento y cuidados

${ }^{30}$ El término "paciente" incorpora un valor cognitivo pasivo que hace referencia a las relaciones asimétricas de poder con respecto a los profesionales de la salud, particularmente el médico. Diferente categoría resulta si se empleara el término "usuario de los servicios" (Mayte Caramés, comentario personal).

${ }^{31}$ Hace ya tiempo que Roberto Campos (1997a y 1997b ) demostró lo pertinente de introducir hamacas en un hospital indígena en México, porque las camas resultaban tremendamente incómodas para los usuarios indígenas. Sabemos que, tanto las infraestructuras como el mobiliario son importantes para la comodidad de los usuarios atendidos o ingresados en una unidad hospitalaria. ¿Las autoridades sanitarias y los arquitectos tendrán a bien escuchar en alguna ocasión, en el diseño de los hospitales, el parecer de los usuarios y sus expectativas? ¿Son compatibles con el pragmatismo de las infraestructuras y sus requerimientos biomédicos?

${ }^{32}$ Particularmente relevante entre los creyentes musulmanes.

${ }^{33}$ Recordemos el rechazo a las transfusiones sanguíneas por parte de algunas confesiones religiosas como los Testigos de Jehová. 
cuando no reflejo de un sectarismo asistencial marcado por el paternalismo. Hace un tiempo cayó en mis manos un folleto, supuestamente basado en criterios desarrollados por "facilitadores culturales", a la manera de un "protocolo hospitalario para inmigrantes", donde se instaba a los inmigrantes de origen marroquí a practicar con regularidad la higiene de todas sus extremidades. Estas consideraciones no hacen justicia con la historia de los que fueron los descubridores de los baños públicos y además estigmatizan al "Otro", confirmando los estereotipos y lugares comunes falsos que se emplean regularmente como arma arrojadiza. El problema de estos "protocolos" de supuesta sensibilidad cultural es que están pensados no para personas en situación de ingreso hospitalario, sino sólo para "inmigrantes". Esta cuestión me va a facilitar la última reflexión con este recorrido de ida y vuelta desde las altiplanicies bolivianas a las extensiones de la Península Ibérica. Hace unos meses se distribuyó por REDAM ${ }^{34}$ una publicidad relativa a la "Declaración de Ámsterdam" basada en una red de "Hospitales Amigos de los Inmigrantes" por toda Europa, con competencia cultural al respecto. Bien está hacer visible un problema y tomar medidas que permitan un mayor nivel de satisfacción de los usuarios de dichos servicios sanitarios; lo que me preocupa es que la loable iniciativa pueda contribuir, sin pretenderlo, a la configuración de guetos sanitarios, donde los usuarios inmigrantes tengan sus respectivos hospitales, como sucedía en tiempos de la Colonia con los "hospitales de indios", circunstancia que iría asociada a la idea de "mala calidad" de los servicios. Si la interculturalidad no es reconocida como un bien patrimonio de la sociedad en su conjunto y, por el contrario, es contemplada como "cosa de inmigrantes" $\mathrm{O}$ "cosas de indios" permitiremos que bajo sus atractivos ropajes de comprensión y tolerancia, nidifiquen la exclusión, el racismo y la xenofobia.

\section{BIBLIOGRAFÍA CITADA}

ABSI, PASCAL. 2005. Las percepciones de las enfermedades por parte de los jóvenes en el barrio minero de San Cristóba, Potosí, Bolivia. La Paz: IPIEB (Inédito).

Aguirre Beltrán, GonZAlo. 1994. Programas de salud en la situación intercultural. Obra Antropológica $V$ [1955] México: FCE/Universidad Veracruzana/Instituto Nacional Indigenista/Gobierno del Estado de Veracruz.

AlBó Corrons, XAVIer. 1995. Bolivia Plurilingüe. Guía para planificadores y educadores. La Paz: CIPCA, 3 vols.

\footnotetext{
${ }^{34}$ Red de Antropología Médica que agrupa a varios profesionales, docentes e investigadores interesados por la Antropología Médica, tanto en España como en América Latina.
} 
- 2004. "Salud e interculturalidad", en G. Fernández (coord.), Salud e interculturalidad en América Latina. Perspectivas antropológicas: 65-74. Quito: Abya-Yala/Bolhispania/ UCLM.

ARnold, Denise y Juan De Dios Yapita. 1999. Vocabulario aymara del parto y de la vida reproductiva de la mujer. La Paz: ILCA/FHI.

CAMPOS NAVARRO, ROBERTO. 1997a. "La hamaca: historia, etnografía y usos médicos de un mueble americano". Revista Médica del IMSS 35 (4): 287-294.

- 1997b "La satisfacción del enfermo hospitalizado: empleo de hamacas en un hospital rural del sureste de Méxicon. Revista Médica del IMSS 35 (4): 265-272.

- 2004. "Experiencias sobre salud intercultural en América Latina", en G. Fernández (coord.), Salud e interculturalidad en América Latina. Perspectivas antropológicas: 129-151. Quito: Abya-Yala/Bolhispania/UCLM.

- y LUCA CITARELLA. 2004. "Willaqkuna. Un programa de formación en salud intercultural en Bolivia", en G. FERNÁNDEZ (coord.), Salud e Interculturalidad en América Latina. Perspectivas antropológicas: 93-106. Quito: Abya-Yala/Bolhispania/UCLM.

Chaumeil, JeAn PierRe. 2004. "Del proyectil al virus. El complejo de dardos - mágicos en el chamanismo del oeste amazónico", en G. FERNÁNDEZ (coord.), Salud e interculturalidad en América Latina. Perspectivas antropológicas: 265-278. Quito: Abya-Yala/Bolhispania/UCLM.

DiBBiTS, INEKE. 1994. Lo que puede el sentimiento. La temática de la salud a partir de un trabajo con mujeres en El Alto Sur. La Paz: Tahipamu.

- y MARISKA DE BOER. 2002. Encuentros con la propia historia. Interculturalidad y Trato Humano desde la perspectiva de un equipo de salud. La Paz: Tahipamu.

- y Magali Terrazas. 2003. Uno de Dos. El involucramiento de los hombres en la atención de la salud perinatal. Revelaciones desde Santa Rosa y Rosas Pampa. El Alto . La Paz: Tahipamu.

DOSSEY, LARRY. 1999.Tiempo, Espacio y Medicina [1982]. Barcelona: Kairós.

FERNÁNDEZ JUÁrEZ, GERARDO. 1999. Médicos y yatiris. Salud e interculturalidad en el altiplano aymara. La Paz: CIPCA/OPS/Ministerio de Salud y Previsión Social.

- 2002. Aymaras de Bolivia. Entre la tradición y el cambio cultural. Quito: Abya-Yala.

- 2004a. Yatiris y ch'amakanis del altiplano aymara. Testimonios, sueños y prácticas ceremoniales. Quito: Abya-Yala.

- 2004b. "Médicos y kharisiris. Dificultades para la aplicación de políticas interculturales en salud en el altiplano aymara de Bolivian. Congreso Internacional de Americanistas de Perugia (en prensa).

- (coord.). 2004. Salud e interculturalidad en América Latina. Perspectivas antropológocas. Quito: Abya-Yala/Bolhispania/UCLM.

FloRes MARTOS, JUAN ANTONO. 2004. “Una etnografía del 'año de provincias' y de 'Cuando no hay doctor'. Perspectivas de salud intercultural en Bolivia desde la biomedicina convencional, en G. FERNÁNDEZ (coord.), Salud e interculturalidad en América Latina. Perspectivas Antropológicas: 181-212. Quito: Abya-Yala/ Bolhispania/UCLM.

FOSTER, GEORGE. 1980. "Relaciones entre la medicina popular española y latinoamericana", en M. Kenny y J. M. de Miguel, La antropología médica en España: 123-147. Barcelona: Anagrama.

GutiérREZ Estévez, MANUEl (ed.). 2000. Sustentos, aflicciones y postrimerias de los indios de América. Madrid: Casa de América.

MEnÉNDeZ, EduARdo. 1981. Poder. Estratificación y salud. México: Ediciones de la Casa Chata. 
- 1992. "Modelo hegemónico, modelo alternativo subordinado, modelo de auto atención, caracteres estructurales", en R. CAMPOS (comp.), La antropología médica en México. México: Instituto Mora-UAM.

Platt, Tristan. 2001. "El feto agresivo. Parto, formación de la persona y mito- historia en los Andes". Anuario de Estudios Americanos LVIII (2).

RANCE, SUSANNA. 1999. Trato bumano y educación médica. Investigación-acción con estudiantes $y$ docentes de la carrera de medicina UMSA, La Paz. La Paz: Viceministerio de Asuntos de Género, Generaciones y Familia.

SEMFYC. 2004. "Influencia en la salud de los factores culturales y sociales de los inmigrantes", El médico interactivo. Diario electrónico de la sanidad.

(www.elmedicointeractivo.com/formacion_acre2004/tema9/inmigrantes3.htm).

YolPAHTLI. 2003. Servicios de salud con calidad intercultural en pueblos indios. México: Secretaría de Salud. 